\title{
Determination of Reserve Capacity under the Regional Interaction Operation with Wind Power Integration
}

\author{
Liu Rao/s per 1st Affiliation \\ School of Electrical Engineering \\ Dalian University of Technology,DLUT \\ Dalian, Liaoning, China \\ raoliu@dlut.edu.cn \\ Zhang Xin/s per 2nd Affiliation \\ School of Electrical Engineering \\ Dalian University of Technology,DLUT \\ Dalian, Liaoning, China \\ zhangxin_dut@163.com \\ Chang Yekui/s per 3th Affiliation \\ School of Electrical Engineering \\ Dalian University of Technology,DLUT \\ Dalian, Liaoning, China \\ changyek@163.com \\ Wang Yan/s per 4st Affiliation \\ School of Electrical Engineering \\ Dalian University of Technology,DLUT \\ Dalian, Liaoning, China \\ wdoudouy@163.com
}

\author{
$\mathrm{Ba} \mathrm{Yu/s} \mathrm{per} \mathrm{5st} \mathrm{Affiliation}$ \\ School of Electrical Engineering \\ Dalian University of Technology,DLUT \\ Dalian, Liaoning, China \\ bayu@mail.dlut.edu.cn
}

Shen Shuo/s per 6st Affiliation

School of Electrical Engineering

Dalian University of Technology,DLUT

Dalian, Liaoning, China

ssay_1314@163.com

\author{
Song Yi Wen/s per 7st Affiliation \\ School of Electrical Engineering \\ Dalian University of Technology,DLUT \\ Dalian, Liaoning, China \\ songyiwen@mail.dlut.edu.cn
}

\section{Li Weidong/s per 8st Affiliation*}

School of Electrical Engineering

Dalian University of Technology,DLUT

Dalian, Liaoning, China wdli@dlut.edu.cn

* Corresponding Author

\section{INTRODUCTION}

Abstract-Starting from the regional interaction operation with wind power integration, based on the generalized area forming from interactive operation, the article propose to take the basic unit of system node as the study object. Then analyze the regional interaction operation's influence on the determination of reserve capacity. By calculating the correlation coefficient between various random time sequences of uncertainties, confirm the overall forecasting error distribution, and then obtain the system reserve capacity according to the reliability index-loss of load probability. To improve the economy effectively, take ILH to involve in sharing reserve capacity, on the one hand, to effectively deal with the uncertainty of the small probability events, on the one hand, to optimize the reserve costs together with traditional reserve. So this can meet reliability and economy of reserve configuration. Therefore aiming at minimizing the total cost of reserve, establish the reserve allocation model to optimize reserve costs.

Keywords- Regional Interaction Operation; Correlation Coefficient; Reserve Capacity; Reliability; Reserve Allocation
On behalf of the most developing scale renewable energy generation[1], wind power gradually attracts more and more attention. The power grid should take various effective ways to accept wind power to take full advantage of clean energy.

Wind power has random, intermittent and fluctuant features, so the large-scale access will result in increasing system reserve capacity, peak load regulating capacity and system operating costs. According to the traditional policy, the renewable energy generation should consume in the province individual by principle, however, it easily results in the lack of reserve capacity causing security risk. Paper [2] proposed the source-net-load interactive operation concept, one element of which is opening tie line plan, allocating wind power to multiple areas to regulate corporately on the premise of ensuring security, and transferring local consumptive to wide coordination. According to the situation of wind power output, the power grid integrates power generation and load nodes suitable for wind power consumption, forming a general 
area of wind power consumption.For different situations of wind fluctuations, the composition of power generation and load nodes suitable for consuming wind power in each control area is different, which changes the boundary of general area dynamically. The change of control area boundary has an impact on reserve capacity certainly, so there is necessary to study how to determine reserve capacity under dynamic boundary varying.

The existing methods to determine reserve capacity consider fixed area boundaries mostly, which are roughly divided into certainty and probability two kinds. The certainty often take a fixed percentage of maximum load or the maximum generator capacity, which considers economic poorly, so the probability is the most common to determine reserve capacity currently. Some studies adopt particle swarm algorithm to optimize based on MonteCarlo simulation technique[3,4]. Paper [5] built the model correlating wind power forecasting error with reserve demand by filtering wind power forecasting data through cluster analysis. Paper [6] constructed the reserve optimization model aiming to minimize the total cost, using genetic algorithms and interior point methods in discrete and continuous field alternately to solve. As the wind power cross-area consuming causes varying the general control area boundary, the methods above need to determine the system parameters, build optimization model and solve the optimal value repeatedly again. The computation process is quite tedious, so the current method can not be effectively applied to the power grid under boundaries varying dynamically.

Furthermore, in the source-gird-load interactive operation, the load side of system is also able to participate in bearing reserve capacity except the generation side. Flexible load (FL) in smart grid will flexibly adjust power consumption according to the current relation between supply and demand to provide reserve support to cope with wind power fluctuation. According to the actual situation, FL gets into or out of control area to stabilize fluctuation, at the same time, it will further promote the area boundaries varying.

This paper proposes to study node and confirm the correlation between uncertain time sequences to acquire the total load forecasting error. Then calculate reserve capacity based on reliability index. To guarantee economic optimal, take flexible load into sharing reserve capacity together with traditional reserve to achieve the minimum total cost.

\section{ANALYSIS OF UNCERTAINTIES}

\section{A. Handle of Uncertainties}

\section{1) Load forecasting error.}

As an important part of power system plan, there will exist errors in load orecasting by influence of weather, holidays, forecasting methods and other factors. The Power plants should arrange power output based on the forecasting value in future, so the forecasting accuracy will directly affect the magnitude of reserve capacity, which is the basis of realizing power grid operation security, quality and economic. The statistical material in $[7,8]$ indicates the random fluctuation of load is normal distribution, the variance of which is related to the accuracy of load forecasting.

\section{2) Wind power forecasting error.}

The wind farm connected to the grid is equivalent to a disturbance ource of grid, which will have an impact on the reliability of of grid operation. So the wind power output in real grid operation is seen as a "negative load".Wind power forecasting is based on the forecast of wind speed, which will vary from the real-time weather conditions, so the forecasting value must exist some errors. Because wind turbines disperse relatively, according to the central-limit theorem, wind power forecasting error can be approximately thought as a normal distribution [9]. System reserve capacity should have enough ability to cope with the wind random fluctuations to maintain the power balance.

\section{B. Analysis of System Uncertainty}

The regional interaction operation directly results in blurring area boundaries and changes the area that the nodes belong to. So this paper studies nodes' dynamic changes' influence on reserve capacity from the perspective of the nature. The uncertainties which have the greatest impact on reserve are the nodes of load and wind power, so we study the power forecasting errors of both nodes, which are seen as normal distribution of random time sequences. We confirm the relationship between these random time sequences. Integrate the variance of each forecasting error together and obtain the total variance of the entire area.

The direct method to calculate the variance of system forecasting error is to add each random time sequence and get the total variance of gained sequence. However, due to the large number of samples of each random time sequence, each change of the boundaries need to repeat calling these sample data groups, which is extremely inconvenient.

In the following method of calculating the correlation coefficient between the random time sequences deriving the total variance of system by the probability theory, we only need to know the characteristic values of each random time sequence, namely expectation and variance, without using the large sample data to get total variance, which is much clearer.

The standard deviation of forecasting error of each node is:

$$
\sigma=\sqrt{\frac{1}{\mathrm{M}} \sum_{i=1}^{\mathrm{M}}\left[\left(P_{\text {meas }, i}-P_{\text {pred }, i}\right)-\left(\overline{P_{\text {meas }}}-\overline{P_{\text {pred }}}\right)\right]^{2}}
$$

Among above, $\mathrm{M}$ is the amount of the historical sample data of forecasting error, $P_{\text {pred }, i}$ is the power forecasting of sample $i, P_{\text {meas }, i}$ is the actual power of sample $i, \overline{P_{\text {pred }}}$ is the average of forecasting power in $\mathrm{M}$ samples, $\overline{P_{\text {meas }}}$ is the average of measures in $\mathrm{M}$ samples.

According to the covariance formula by the theory of probability, we can get:

$$
\operatorname{Cov}\left(\Delta P_{x}, \Delta P_{y}\right)=\frac{1}{\mathrm{M}} \sum_{i=1}^{\mathrm{M}}\left(\Delta P_{x, i}-\overline{\Delta P_{x}}\right)\left(\Delta P_{y, i}-\overline{\Delta P_{y}}\right)
$$


The correlation coefficient between each forecasting error is:

$$
r_{x y}=\frac{\operatorname{Cov}\left(\Delta P_{x}, \Delta P_{y}\right)}{\sigma_{x} \sigma_{y}}=\frac{1}{\mathrm{M}} \frac{1}{\sigma_{x} \sigma_{y}} \sum_{i=1}^{\mathrm{M}}\left(\Delta P_{x, i}-\overline{\Delta P_{x}}\right)\left(\Delta P_{y, i}-\overline{\Delta P_{y}}\right)
$$

The correlation coefficient value $r_{x y}$ is between -1 and 1 , which is a measure of the linear relationship between the variables portray the $\Delta P_{x}$ and $\Delta P_{y}$.

If the correlation coefficient is positive, the superposition of each error will cause the total error distribution curve showing violent fluctuations, which increases the volatility of uncertainties. The negative coefficient between factors is an important element in smoothing uncertainty fluctuations, which causes the overall error distribution curve showing the effect of smoothing, namely peak load shifting.

Based on the correlation coefficient of each time sequence, the total variance is:

$$
\sigma_{\text {sum }}^{2}=\left[\begin{array}{llll}
\sigma_{1} & \sigma_{2} & \ldots & \sigma_{\mathrm{N}}
\end{array}\right]\left[\begin{array}{cccc}
r_{11} & r_{12} & \ldots & r_{1 \mathrm{~N}} \\
r_{21} & r_{22} & \ldots & r_{2 \mathrm{~N}} \\
\ldots & \ldots & \ldots & \ldots \\
r_{\mathrm{N} 1} & r_{\mathrm{N} 2} & \ldots & r_{\mathrm{NN}}
\end{array}\right]\left[\begin{array}{c}
\sigma_{1} \\
\sigma_{2} \\
\ldots \\
\sigma_{\mathrm{N}}
\end{array}\right]
$$

Among above, $\mathrm{N}$ is the amount of random time sequences.

This method takes the correlation of various sequences into account and reduces the total standard deviation of forecasting error through smoothing effect. The uncertainty fluctuations offset fully and we can easily judge the effect on system operation by checking the correlation coefficient value is positive or negative. On the other hand, when the control area boundary changes with the operation of system, the number of nodes in which will also increase or decrease. Correlation coefficient between each time sequence can be calculated in advance and will not change with boundary. So there is only need to change linearly the degree of Equation (4) to get the overall standard deviation, which isn't required to consider the entire system operation to calculate iteratively, significantly reducing the amount of calculation, which has more obvious advantages in the regional interaction operation of smart grid.

\section{DETERMINATION OF THE SYSTEM RESERVE CAPACITY}

As an uncontrollable power generation, wind power is often considered as a "negative load". Superimpose the wind power and load forecasting error to get the total load forecasting error density curve, as shown the solid line in Fig.1, which is still a normal distribution. The horizontal axis represents the forecasting error, the vertical axis represents the probability of the occurrence of load errors.

Take $P_{\text {LOLP }}$ as system reliability index, that is the probability of generating capacity not meeting the system load demand. If we take the reserve capacity for $P_{2}$, the reserve at the moment is not sufficient to cope with the load fluctuation of the right shadow, then loss of load probability is the proportion of the right side shadow.

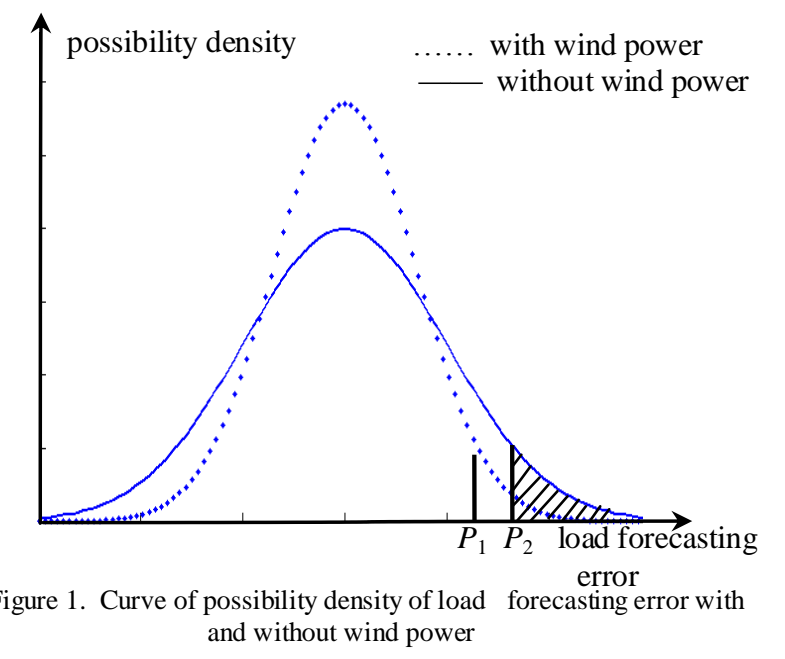

If $P_{\mathrm{LOLP}}$ is known, which is the shaded area of the curve tail, calculate the standard deviation osum of total forecasting error by the method of correlation coefficient. We can find the reserve capacity by the nature of curve of normal distribution, so the reserve capacity is calculated as follows:

$$
P_{\text {reserve }}=\sigma_{\text {sum }} \phi^{-1}\left(1-P_{\mathrm{LOLP}}\right)+\mu_{\text {sum }}
$$

Among above, $\phi^{-1}$ is the inverse function of standard normal distribution, $\mu_{\text {sum }}$ is the expectation of total load forecasting error sequence, $\mu_{\text {sum }}=\mu_{1}+\mu_{2}+\cdots+\mu_{\mathrm{N}}$.

When the wind power doesn't participate in the power grid, reserve capacity only needs to deal with the uncertainties of load fluctuation. Because the wind power forecasting errors add into the system joining with wind power, the variance of load forecasting error will be greater, as shown in Fig .1. Under the circumstances ensuring the same reliability of power grid, it is bound to increase a certain number of reserve capacity in response to the influence of wind power fluctuations to system. Visually seen from Fig .1, when the shadow area is the same, namely having the same reliability, the reserve demanded increases from $P_{1}$ to $P_{2}$.

\section{FL INVOLVING IN RESERVE SHARING}

\section{A. Necessity of Introducing FL}

As seen in Fig .1, the tail of load forecasting error density curve with wind power becomes longer, which indicates high-risk events occurring more frequently. If all the reserve is assumed by generation side, it should be reserved regardless of whether invoked, which is very uneconomical. So it isn't worth for low-possibility reserve of tail, we consider using probabilistic reserve to cope with high-risk and small probability event.

Reserve of the demand side has a considerable advantage over traditional in dealing with small probability event, because users can interrupt the contract and adjust its power according to the power generation and load balance relation [10]. The load which can act as reserve is called Interruptible Load (IL). IL is divided into 
interruptible load with low price (ILL) and interruptible load with high compensation (ILH), the difference between which is the way of compensation. ILL is compensated by price discounts before called, and ILH is by high prices after called. That is, no matter whether ILL is called, there is need to pay the same fees.

But ILH is different. If ILH isn't called, power companies don't need to pay the capacity cost. Only is called, pay a higher amount of compensation. Because when the probability of called is low, which multiplying compensation may be lower than the cost of traditional reserve and ILL, ILH is more applicable to the case of small probability. Therefore based on its own characteristics, as demand-side reserve, ILH can well support for the system reserve, which can effectively reduce reserve costs and improve the flexibility of system.

\section{B. Reserve Costs Allocation}

\section{1) Traditional reserve cost}

Power companies need to buy reserve service from power plants, whose costs often include the capacity cost and electricity cost two parts [11].

Capacity cost aims at the reserve from generator which must be set aside, regardless of which is called, we are required to pay for the opportunity cost of the part of reserve.

Capacity cost can be expressed as:

$$
C_{t r}=Q_{t} P_{r} t
$$

Among above, $Q_{\mathrm{t}}$ is the traditional reserve set aside, $P_{\mathrm{r}}$ is the price of capacity.

Electricity cost need consider the probability of reserve capacity to be called. If the reserve capacity is actually called, we need to pay the cost of this part.

Electricity cost can be expressed as:

$$
C_{t d}=\sum_{i=1}^{m} \Delta L_{i} P_{i} P_{d} t
$$

Among above, $P_{\mathrm{d}}$ is the price of electricity, $m$ is the number of segments of traditional reserve capacity, $\Delta L_{i}$ is the capacity of segment $i$ called, $\Delta L_{1}+\Delta L_{2}+\cdots \Delta L_{m}=Q_{t}, P_{i}$ is the possibility of segment $i$ called.

\section{2) ILH cost}

As load-side reserve, ILH participates in operating power grid actively to cope with small probability event, which decreases its capacity in short time to maintain the power balance.

The cost of ILH is:

$$
C_{\mathrm{ILH}}=Q_{\mathrm{ILH}} P_{\mathrm{ILH}} t
$$

Among above, $Q_{\mathrm{ILH}}$ is the outrage capacity of ILH, $P_{\mathrm{ILH}}$ is the price of ILH.

\section{3) Allocation principle}

After the wind power involves in the power grid, in order to achieve the best economic, we can establish the appropriate allocation model for reserve cost.

$$
\begin{gathered}
\min C=C_{\mathrm{tr}}\left(Q_{\mathrm{t}}\right)+C_{\mathrm{td}}\left(Q_{\mathrm{t}}\right)+C_{\mathrm{ILH}}\left(Q_{\mathrm{ILH}}\right) \\
\text { s.t. } \quad Q_{\mathrm{t}}+Q_{\mathrm{ILH}}=P_{2} \\
0 \leq Q_{\mathrm{t}} \leq P_{2} \\
0 \leq Q_{\mathrm{ILH}} \leq P_{2}
\end{gathered}
$$

By solving this model, we can determine the traditional and ILH capacity correspondingly.

\section{ANALYSIS OF EXAMPLES}

\section{A. Calculation of Reserve Capacity}

1) Calculation of reserve capacity by correlation coefficient

The control area in this example contains 5 load nodes and 4 wind power nodes. The time sequences of wind power and load forecasting error are normally distribution, whose numerical characteristics as shown in Tab .1.

TABLE 1 Characteristics of the forecasting error of 9 nodes

\begin{tabular}{|c|c|c|c|c|c|}
\hline Node(Load) & 1 & 2 & 3 & 4 & 5 \\
\hline $\begin{array}{c}\text { Expectation } \\
\text { (p.u.) }\end{array}$ & 3 & 5 & -3 & -6 & -4 \\
\hline $\begin{array}{c}\text { Standard } \\
\text { Deviation }\end{array}$ & 0.8 & 0.5 & 1.1 & 1.8 & 1.7 \\
\hline $\begin{array}{c}\text { Node } \\
\text { (Wind) }\end{array}$ & 6 & 7 & 8 & 9 & \\
\hline $\begin{array}{c}\text { Expectation } \\
\text { (p.u.) }\end{array}$ & 4 & 2 & 1 & -2 & \\
\hline $\begin{array}{c}\text { Standard } \\
\text { Deviation }\end{array}$ & 1.5 & 0.8 & 0.4 & 1.9 & \\
\hline
\end{tabular}

Correlation coefficient between the time sequences of uncertainty factors as follows:

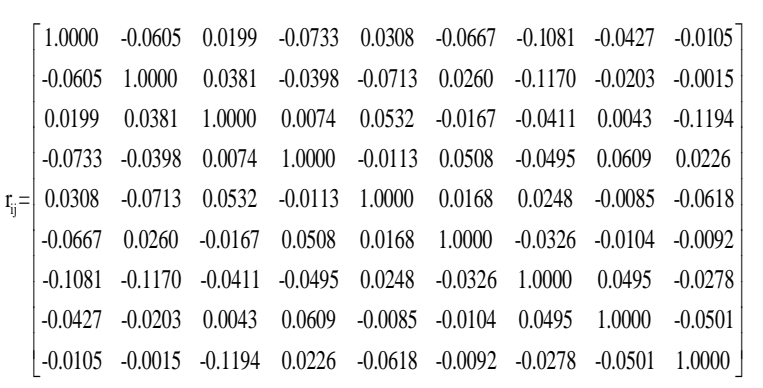

The correlation coefficient between two same time sequences is 1 , and the closer the correlation coefficient approches 1 , the more benefit to smoothing fluctuations the superposition of both has, which reduced the reserve requirements of the system to a certain extent.

According to the standard deviation of each random time sequence and the correlation coefficient between each other, we can get the standard deviation of $\sigma_{\text {sum }}=3.6590$. Based on the reliability index, namely $P_{\mathrm{LOLP}}=1.3 \%$. The reserve capacity of the area is $P=8.3952$ by calculating from reserve formula.

2) Calculation of reserve capacity by adding directly

In order to verify the correlation coefficient method reasonable and accurate, we adopt the most primitive method of adding directly to verify. Add the random time sequences of all uncertain factors directly to get the distribution curve of the system total forecasting error. In 
the condition of $P_{\mathrm{LOLP}}$ still being $1.3 \%$, calculate the required reserve capacity $P=8.3952$.

The result is the same as that gotten from the correlation coefficient method, which proves the accuracy of the correlation coefficient method to calculate reserve capacity.

\section{B. Reserve Capacity Allocation}

In the previous chapter we've acquired the total reserve capacity is 8.3952. In the model, capacity price $P_{\mathrm{r}}$ is $113 \$ /$ p.u., electricity price $P_{\mathrm{d}}$ is $280 \$ /$ p.u.. ILH adopts segmented prices, shown in Tab .2 , in which the price increases with capacity.

TABLE 2 Segmented prices of ILH

\begin{tabular}{|c|c|}
\hline ILH Capacity $Q_{\mathrm{IL}}$ (p.u.) & ILH Price $P_{\mathrm{IL}}(\$)$ \\
\hline$Q_{\mathrm{IL}} \leq 3$ & 400 \\
\hline $3<Q_{\mathrm{IL}} \leq 6$ & 700 \\
\hline$Q_{\mathrm{IL}}>6$ & 1000 \\
\hline
\end{tabular}

Calculate how to share the reserve through traditional reserve and ILH. In the process of Simulink, we record the total costs by changing the proportion of traditional reserve. Plot as Fig.2.

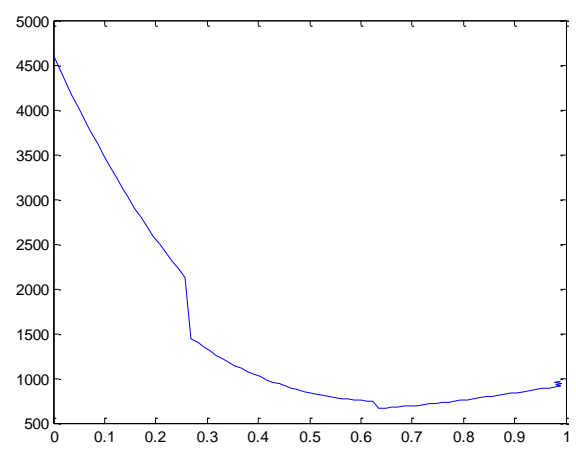

Figure 2. Curve of total cost

We can see from the diagram, if the proportion of traditional reserve to total reserve is 1 , that is, all the reserve is bared by traditional, the total cost is $949.3213 \$$. Along with the proportion changing, the cost curve appeared a low point. At this moment, the reserve cost is least. The best result of sharing reserve cost is shown in Tab .3.

TABLE 3 Result of sharing reserve cost

\begin{tabular}{|c|c|c|}
\hline $\begin{array}{c}\text { Traditional Reserve } \\
\text { Capacity (p.u.) }\end{array}$ & $\begin{array}{c}\text { ILH Capacity } \\
\text { (p.u.) }\end{array}$ & $\begin{array}{c}\text { Total Reserve } \\
\text { Capacity(p.u.) }\end{array}$ \\
\hline 5.4000 & 2.9952 & 8.3952 \\
\hline $\begin{array}{c}\text { Traditional Reserve } \\
\text { Cost(\$) }\end{array}$ & $\begin{array}{c}\text { ILH Cost } \\
(\$)\end{array}$ & $\begin{array}{c}\text { Total Cost } \\
(\$)\end{array}$ \\
\hline 611.4532 & 79.8192 & 691.2724 \\
\hline
\end{tabular}

By comparison, adopting the method of sharing reserve reduces the costs from $949.3213 \$$ to $691.2724 \$$, which well achieves the optimal economy of the system.

\section{CONCLUSIONS}

This paper proposed the method of calculating correlation coefficient between various random time sequences of forecasting error between nodes' uncertainties to obtained the system load forecasting error distribution, then get the desired reserve capacity by the error distribution curve. Today large-scale renewable energy access and interactive operation is more prevalent. The area boundaries become blurred and those between areas are no longer so clear. This method can be a good way to deal with variable area boundaries and under the existing conditions we can quickly and accurately obtain the reserve capacity, which is more practical.

By ensuring the reliability index $P_{\text {LOLP }}$ same, we determine the reserve capacity to guarantee reliability. With ILH sharing reserve capacity, we can reduce the reserve costs to achieve the purpose of optimal economic. So the method we adopt take into account the reliability and economy of system.

\section{ACKNOWLEDGMENT}

This work was supported by the State Grid Corporation of China Project: "Study on Key Technologies for Power and Frequency Control of System with Source-Grid-Load Interactions".

\section{REFERENCES}

[1] Weidong Chou, Zhidong Wang, Juan Li. Analysis of wind power development 's problems in our country [J]. Electric Power Technologic Economics,2008,20(1):19-23.

[2] Jianguo Yao, Shengchun Yang, Ke Wang, et al. " source- gridload" interactive operation control concept and research framework of smart grid [J]. Power System Automation,2012,36(21):1-6.

[3] Danping Wang, Zhixiang Chen, Mengfu Tu, et al. Reserve capacity calculation considering large scale wind power [J]. Power System Automation,2012,36(21):24-28.

[4] Chunying Lin. Optimization research based on GA-IPM for spinning reserve containing wind power system [D]. Guangxi University,2013.

[5] Min Yu, Minchen Yang, Zhuanwen Jiang, et al. Research of power system reliability assessment and reserve optimization involving wind power $[\mathrm{J}]$. Power System Protection and Control,2012,40(12):100-104,135.

[6] Tianyu Xia. Research of reserve scheduling in regional power grid with high penetration of wind power [D]. Harbin Institute of Technology,2012.

[7] Lunnong Tan, Baohui Zhang. Emergency reserve capacity under market environment [J]. Proceedings of the CSEE,2002,22(11):5458.

[8] Baohui Zhang, Liyong Wang, Lunnong Tan, et al. Auxiliary Services trading mechanisms based on the reliability of supply and demand both sides bid $[\mathrm{J}]$. Power System Technology,2005,29(23):50-55.

[9] Pinson P. Estimation of the uncertainty in wind power forecasting $[\mathrm{J}] .2006$.

[10] Weiqing Sun, Chengmin Wang, Yan Zhang. Flexible load of smart grid [J]. Power Demand Side Management,2012(3):10-13.

[11] Shuangxi Zhou, Haichao Wang, Shousun Chen. Operation value analysis of wind power $[\mathrm{J}]$. Power System Technology,2006,30(14):98-102. 